\section{Uniaxial Wireless Tissue Palpation Device for Minimally Invasive Surgery}

\section{Marco Beccani}

\section{Christian Di Natali}

Vanderbilt University,

Nashville, TN 37235

\author{
Mark Rentschler \\ University of Colorado, \\ Boulder, CO 80309
}

\section{Pietro Valdastri \\ Vanderbilt University, \\ Nashville, TN 37235}

\section{Background}

Nowadays surgery tends to be performed with minimally invasive techniques that result in less post-operative pain, recovery time and smaller incisions. However, unlike open surgery, during minimally invasive surgery (MIS) the surgeon has few chances to rely on tactile and kinesthetic feedback [1]. Tissue palpation aids in preventing traumas or in leveraging tactile information to identify tumors, vessels and organic structures buried underneath the tissue surface. Therefore, in the last two decades, research on palpation devices strived to restore tactile and kinesthetic sensations in MIS. This was pursued by performing tissue palpation with different techniques, such as indenting [2], grasping [3] or rolling [1]. Despite this research effort, a translation into clinical practice is still far to come. Even further, surgeons are resistant to the option of devoting a surgical port to a palpation device, because of the available ports are needed for the MIS instruments. As an alternative to this approach, intraoperative ultrasonography devices (IOUS) may be used to detect buried structures [4]. Also this technology, however, requires a dedicated surgical port during the entire procedure.

\section{Method}

In this paper, we study the feasibility of a wireless approach to palpation for a single degree of freedom. In the proposed approach, a wireless tissue palpation device (WTPD) can be inserted through a standard trocar into the human abdominal cavity, placed by the surgeon on the target region, and then used without taking up a surgical port. The WTPD is a magnetic device that can perform tissue indentation thanks to the resulting intermagnetic force $F_{T}(t)$ between a miniaturized permanent magnet (i.e., embedded inside the WTPD with custom electronic) and an external magnet outside the human body cavity (i.e., fixed at the end effector of a robotic arm). In particular, the kinesthetic data of the tissue under test can be derived from the pressure exerted by the WTPD on the target region and its indentation depth $\delta(t)$. Given the WTPD surface and its weight, the applied pressure can be derived from the force $F_{T}(t)$ that is measured by a load cell mounted in between the magnet and the robotic arm. Gravity force can be removed as an offset. Referring to Fig. 1, when the distance $d(t)$ between the two magnets changes, the indentation depth $\delta(t)$, exerted by the WTPD on the target region, can be derived measuring the $\mathrm{Z}$ component of the magnetic field $B_{z}(t)$ with a

Manuscript received March 15, 2013; final manuscript received April 25, 2013 ; published online June 12, 2013. Editor: Arthur G. Erdman. single axis Hall Effect sensor embedded in the WTPD. As the robotic arm movement is constrained along the vertical direction, the resulting motion of the capsule is also confined along the same axis due to the magnetic coupling. Any misalignment of the device with respect to the $\mathrm{Z}$ axis can be measured and factored out with an embedded triaxial accelerometer (i.e., used as inclinometer) embedded inside the WTPD. Thus $B_{z}(t)$ can be expressed with a univocal function $\Phi[d(t)]$ of the inter-magnetic distance if the magnetic field $B_{z}(t)$ is known for any instant of time it is possible, by inverting $\Phi[d(t)]$, to calculate $d(t)$. $\Phi^{-1}$ was evaluated by fixing the WTPD to a rigid aluminum panel and then measuring $B_{z}(t)$ in a range of $150 \mathrm{~mm}$ from the contact between the magnet holder and the panel (i.e., when this distance is $150 \mathrm{~mm}$ the capsule is at equilibrium with gravity). Five loading unloading cycles were performed by moving the robotic arm at constant speed (i.e., $3.12 \mathrm{~mm} / \mathrm{s}$ ), the data were then fitted with a fifth order polynomial $\left(R^{2}=0.998\right)$ that was used as a calibration function for the device.

Referring to Fig. 1, it is possible to write the expression of distance between the two magnets as a function of time:

$$
d(t)=d\left(t_{0}\right)-\delta(t)-d_{R}\left(t_{0}, t\right)
$$

where $d_{R}\left(t_{0}, t\right)$ is the distance covered by the robotic arm, provided by the robot encoders. Then, taking into account the calibration function of the device $\Phi^{-1}$ and rearranging the terms, we can express $\delta(t)$ with the equation:

$$
\delta(t)=\Phi^{-1}\left[B\left(t_{0}\right)\right]-\Phi^{-1}[B(t)]-d_{R}\left(t_{0}, t\right)
$$

Which provides the indentation depth at a given instant of time. The developed platform is presented in Figs. 2(a) and 2(b). It mainly consisted of the WTPD and the external platform to operate it. The WTPD as shown in Fig. 2(a) contains a $11 \mathrm{~mm}$ by $11 \mathrm{~mm}$ diameter cylindrical $\mathrm{NdFeB}$ magnet (K\&J Magnetics), a 3D MEMS accelerometer (LIS331AL, ST Microelectronics), a Hall Effect sensor (CYP15A, Chen Yang Technologies), a LiPo battery $8 \mathrm{~mm}$ by $15 \mathrm{~mm}$ by $3 \mathrm{~mm}$ (Shenzhen Hondark), a 16 bits ADC (ADS320, Texas Instr.), and a CC2530 wireless microcontroller (Texas Instr.). All these components have been embedded in a cylindrical shell (i.e., $12.7 \mathrm{~mm}$ in diameter, $27.5 \mathrm{~mm}$ in length) that was fabricated with a 3D printer (Objet Geometries LTD) using a not biocompatible material (Vero White, tensile strength $49.9 \mathrm{MPa}$ ). The WTPD weights $117 \mathrm{mN}$ (i.e., $12 \mathrm{~g}$ ) and the probing surface is $113 \mathrm{~mm}^{2}$.

The external part of the platform is presented in Fig. 2(b) and consisted of a 6 DOF robotic arm RV6SDL, Mitsubishi), a 6-axis load cell (MINI 45, ATI, $1 / 16 \mathrm{~N}$ resolution) and a $50 \mathrm{~mm}$ by $50 \mathrm{~mm}$ cylindrical magnet (K\&J Magnetics). The acquired data were transmitted by the microcontroller to an external one and then saved into a PC synchronously to the load cell and the robot position. The sample consisted in a PVC sample (Liquid Plastic, M-F Manufacturing) with a thickness of $34 \mathrm{~mm}$ and elastic modulus of $64.49 \mathrm{kPa}$.

\section{Results}

A prototype implementing this novel palpation device has been tested, confirming the working principle. In particular the indentation depth estimated in Eq. 3 has been validated on the tissue sample using an optical conoscopic holography sensor (Conoprobe, Optimet, USA) adopted as a reference system (resolution $1 \mu \mathrm{m}$ ). The error between the presented approach and the conoprobe is below $0.13 \mathrm{~mm}$, resulting in about $10 \%$ of the full scale range. The WTPD was then compared with a traditional indenter to measure the elastic modulus of the sample. A traditional cylindrical indenter of the same diameter of the WTPD was mounted at the robotic arm end effector replacing the magnet holder. Stress-strain plots are presented in Fig. 3. Elastic moduli obtained by interpolating the data with least square fitting for the traditional 


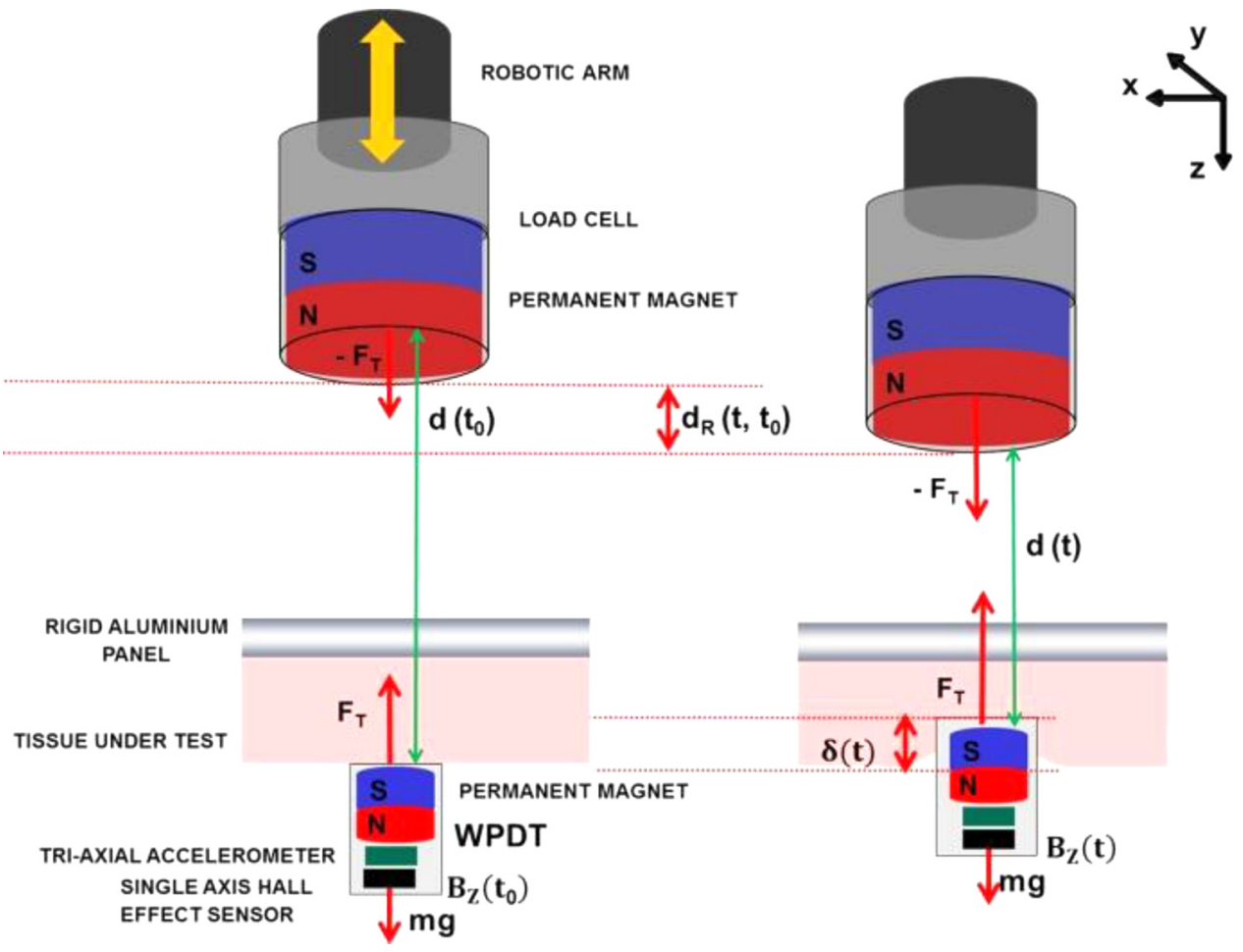

Fig. 1 Principle of operation of the WPTD for the initial instant of time (left) and a generic instant of time (right)

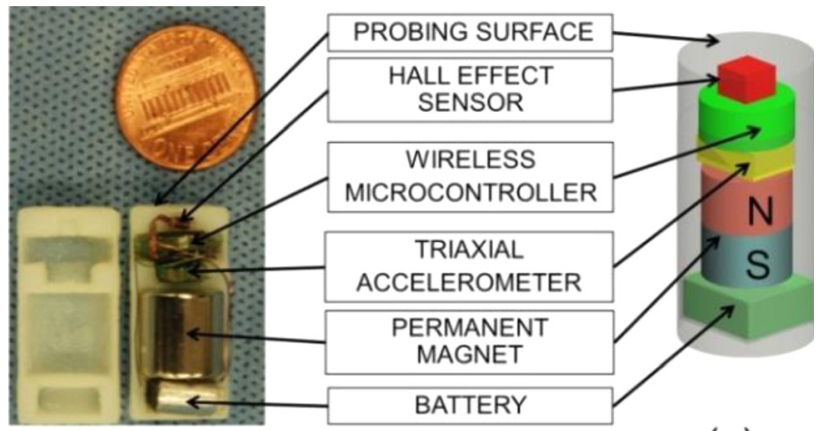

(a)

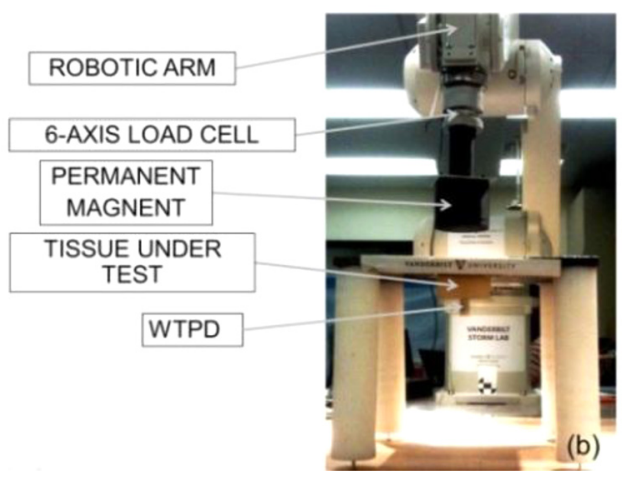

Fig. 2 Picture and schematic view of the wireless tissue palpation device (a); the developed robotic platform (b)

indenter and for the WTPD were respectively $E_{\mathrm{TRAD}}=63.852 \mathrm{kPa}$ $\left(R^{2}=0.992\right)$ and $E_{\mathrm{WTPD}}=64.784\left(R^{2}=0.996\right)$, the average relative error was equal to $1.45 \%$. The accelerometer data confirmed that the motion was always occurring along the $\mathrm{Z}$ direction. Indentation depth reached $1.22 \mathrm{~mm}$ with an indentation force equal to $1.2 \mathrm{~N}$.

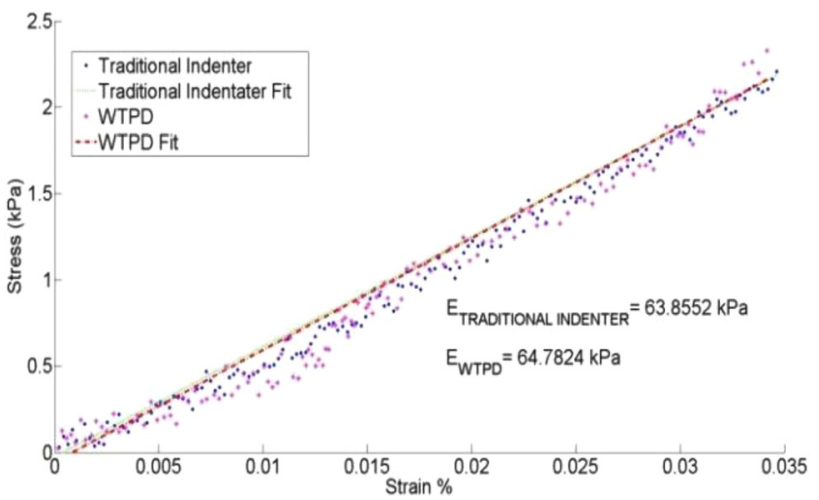

Fig. 3 Experimental data acquired by the traditional indenter and by the WTPD

\section{Interpretation}

So far the research in MIS has focused on restoring tactile and kinesthetic feedback by developing surgical tools which require a mechanical link between the external platform and the target region. Our results showed that wireless tissue palpation is possible for a single degree of freedom on a bench top setting. In these conditions, the WTPD was able to detect the elastic module of a tissue simulator with a negligible error. Extending this result to multiple degrees of freedom and, eventually, to a clinical setting will pose several challenges. First, tridimensional localization will be required to detect deviation from axial motion. The ability of manipulating the capsule to scan the target organ surface will also be crucial. A possible solution consists in rolling indentation [1] that can be obtained by embedding a diametrical magnet into a ball bearing core at the center of the device. Furthermore, in the current platform there is no closed loop control of the magnetic field to set a priori the desired indentation depth as well as a control of the exerted force on the tissue to prevent accidental damages. To better measure and control the indenting force, a 
tactile sensor can be integrated on the probing surface of the WTPD. Finally, external magnetic actuation can be replaced by direct control of the capsule by the surgeon that can grasp a part of the device with a surgical tool and directly indent the tissue.

\section{References}

[1] Liu, H., Li, J., Song, X., Seneviratne, L., and Althoefer, K., 2011, "Rolling Indentation Probe for Tissue Abnormality Identification During Minimally Invasive Surgery," IEEE Trans. Robot., 27(3), pp. 450-460.
[2] Lister, K., Gao, Z., and Desai, J., 2011, "Development of In Vivo Constitutive Models for Liver: Application to Surgical Simulation," Ann. Biomed. Eng., 39, pp. 1060-1073.

[3] Rosen, J., Brown, J., De, S., Sinanan, M., and Hannaford, B., 2008, "Biomechanical Properties of Abdominal Organs In Vivo and Postmortem Under Compression Loads,” J. Biomech. Eng., 130(021020), pp. $1-17$.

[4] Schneider, C. M., Peng, P. D., Taylor, R. H., Dachs, G. W., Hasser, C. J., Di Maio, S. P., and Choti, M. A., 2012, "Robot-Assisted Laparoscopic Ultrasonography for Hepatic Surgery," Surgery, 151(5), pp. $756-762$. 